\title{
1 Die Herkulanischen Papyri und neue Techniken - eine Kurzeinführung
}

Am Vesuv wurden im 18. Jahrhundert etwa tausend antike Buchrollen gefunden. Ihre Lektüre ist ein formidables Puzzle. Doch moderne Methoden entlocken ihnen immer mehr.

Frankfurter Allgemeine Sonntagszeitung $(2021)^{1}$

Die Papyri von Herkulaneum repräsentieren die einzig erhaltene Bibliothek der Antike. Durch den Ausbruch des Vesuvs wurden wie in einer Zeitkapsel hunderte Papyrusrollen karbonisiert und konserviert. ${ }^{2}$ Ihr Inhalt ist für die Altertumswissenschaften, Philosophie, aber auch Theologie von außerordentlichem Wert. Die bis heute fortdauernde Entrollung, Entzifferung, Edition und Einordnung dieser Papyri darf wohl als eines der faszinierendsten und auch anspruchsvollsten Kapitel der Klassischen Philologie gelten. Der junge Friedrich Nietzsche vermerkte einst: ${ }^{3}$ Quanta diligentia, quanta sedulitate volumina Herculanensia in lucem proferantur, nimis est notum quam meis indigeat laudibus. Die Sorgfalt und der Eifer sind bis heute ungebrochen, wenngleich die Papyri in der wissenschaftlichen Wahrnehmung nicht mehr allenthalben so präsent sind wie zu Nietzsches Zeiten. Die meisten Rollen beinhalten griechische Werke epikureisch-philosophischen Charakters, aber auch einige stoische und lateinische Schriften sind zu verzeichnen. Ein Großteil der bisher gefundenen Buchrollen gehörte zur Bibliothek des Epikureers Philodem von Gadara, welcher mit Vergil und anderen augusteischen Dichtern in freundschaftlichem Austausch stand, nicht zuletzt Cicero bekannt war und die Patronage von Cäsars Schwiegervater Piso genoss. ${ }^{4}$

Vielfach wird heute in der Gelehrtenwelt stillschweigend davon ausgegangen, dass die Papyri über 250 Jahre nach ihrer Entdeckung im Jahre 1752 mehr oder weniger erschöpfend erschlossen sind und die Texte nur noch in unbedeutenden Details verbessert werden können. Diese Auffassung ist nichts weniger

1 Ulf von Rauchhaupt, „Verkohlte Wörter,“ Frankfurter Allgemeine Sonntagszeitung (7.2.2021): (54-55) 54.

2 Die inventarisierten Stücke der Herkulanischen Sammlung stammen von etwa 650 bis 1100 Rollen, vgl. Mario Capasso, Volumen. Aspetti della tipologia del rotolo librario antico (Neapel, 1995), 82 und Sergio Carrelli, „Un nuovo punto di vista sulla consistenza della collezione dei papiri ercolanesi,“ CErc 15 (2016): (127-136).

3 Friedrich Nietzsche, Werke und Briefe (Band 4: Schriften der Studenten-und Militärzeit 18661868) (hg. von Hans Joachim Mette und Karl Schlechta; München, 1937), 492.

4 Testimonien-Sammlung zu Philodem bei David Sider, The Epigrams of Philodemos (Oxford, 1997), 227-234 (noch ohne die Passagen am Ende des Index Academicorum). 
als ein kompletter Trugschluss. Einerseits wurden einige bereits aufgerollte Papyri noch niemals ediert und viele Papyri liegen nur in so defizitären Ausgaben vor, dass Neueditionen - anders als bei durch mittelalterlich-byzantinische Handschriften auf uns gekommenen Texten - oftmals einen dramatisch veränderten Text mit zahlreichen Neuinformationen bieten. Andererseits harren heute noch hunderte (!) ungeöffnete Papyrusrollen in der Biblioteca Nazionale di Napoli ihrer Aufwicklung und eine weitaus höhere Zahl könnte noch unter Vulkangestein in der Villa dei papiri in Herkulaneum begraben liegen, welche in großen Teilen unerschlossen ist. Die Gründe für das erhebliche Verbesserungspotential früherer Ausgaben sind vielschichtig, insbesondere sind die verkohlten Papyri aus Herkulaneum mit ihrer kohlebasierten Tinte an vielen Stellen sehr schwierig zu lesen. Die Schwarzschattierungen und Brechungen des natürlichen Lichtes gaukeln dem Leser - also früheren Abzeichnern der Papyri und auch heutigen Gelehrten - bisweilen Buchstaben oder Tintenreste vor, die im nächsten Moment wie eine Fata Morgana wieder entschwinden oder sich zu anderen Buchstaben ändern. Häufig ist es unmöglich, etwas zu erkennen oder die Tinte mit Sicherheit vom Hintergrund zu unterscheiden.

In den letzten Jahren hat die Anwendung innovativer Techniken und neuer philologischer Editionsverfahren die Forschung an den Herkulanischen Papyri geradezu revolutioniert und ungeahnte Perspektiven eröffnet. Es sind prinzipiell zwei Arten von Techniken oder Ansätzen zu unterscheiden: Zum einen wurden und werden bereits entrollte Papyri durch neue Bildgebungsverfahren besser lesbar gemacht, was auch bedeutet, dass auf den Bildern Buchstaben oder Tintenreste zu sehen sind, die für das menschliche Auge (am Original) unsichtbar sind. Zum anderen sind seit geraumer Zeit Bemühungen mit immer vielversprechenderen Zwischenergebnissen im Gange, welche zum Ziel haben, die bisher ungeöffneten Rollen $\mathrm{zu}$ „durchröntgen“ und virtuell aufzuwickeln ohne sie physikalisch entrollen zu müssen. Sollte dieses virtual unrolling in den nächsten Jahren gelingen und ausgereift sein, könnten mehr oder weniger „über Nacht“ hunderte bisher unbekannte, relativ frühe antike Texte zum Vorschein kommen, die wohl über epikureische Spezialität hinaus der Klassischen Philologie und anderen Disziplinen wertvolle Impulse geben würden. 

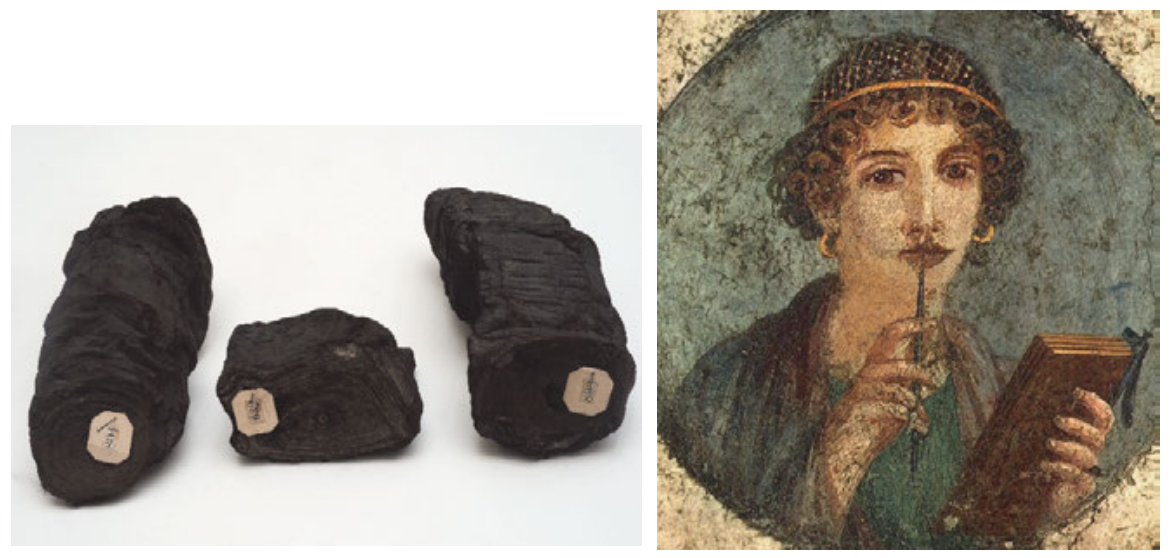

Abb. 1: Drei karbonisierte, ungeöffnete Rol-

Abb. 2: Fresko aus Pompeji („Sappho“) len(teile) aus Herkulaneum

In diesem Büchlein, welches auch als erste deutsche Kurzeinführung in die Herkulanische Papyrologie dienen soll, werden die jüngsten technischen und philologisch-editorischen Entwicklungen sowie neueste Bildgebungsverfahren vorgestellt, die gegenwärtig an den Papyri erprobt werden. Darüber hinaus wird die mit dem Fund der Papyri und Herkulaneum verbundene Kulturgeschichte und Philologie-Geschichte kurz umrissen sowie das textuelle Potential schon aufgerollter und noch unaufgerollter Papyri herausgearbeitet. Ferner wird ein kurzer Einblick in die eigentliche philologische Puzzlearbeit eines Herkulanischen Papyrologen gewährt. Ein unter vielerlei Aspekten sehr bemerkenswerter Papyrus der Herkulanischen Sammlung, Philodems „Geschichte der Akademie“ (Index Academicorum) erfährt eine etwas eingehendere Besprechung und fungiert als Exempel und Bezugspunkt für den Nutzen neuer Techniken und philologischer Methoden - ein Papyrus, der in den letzen Jahren unverhofft viele neue Informationen preisgab. Der Leser möge diese Kurzeinführung als „Protreptikos“ für das Studium der Herkulanischen Papyri betrachten und bei gewecktem Interesse ausführlichere Einführungen, Überblicke und Einzelabhandlungen zu den Papyri, der Villa und Herkulaneum konsultieren. ${ }^{5}$

5 Insbesondere sind zu nennen: Mario Capasso, Manuale di Papirologia Ercolanese (Galatina, 1991); Michael Erler, Epikur - Die Schule Epikurs - Lukrez, in Grundriss der Geschichte der Philosophie. Die Philosophie der Antike, 4. Die hellenistische Philosophie (begründet von Friedrich Ueberweg, völlig neu bearbeitete Ausgabe, hg. von Hellmut Flashar; Basel, 1994); David Sider, 
Die (Herkulanischen) Papyrologen können mit einigem Recht als die „Archäologen der Klassischen Philologie“ bezeichnet werden: Sie graben durch philologisch-kombinatorische Arbeit, sehr eng am physischen Schriftträger, verschüttete Texte hervor und versorgen so die Altertumswissenschaften mit frischem literarischem Rohstoff. Die Editionsarbeit der Papyrologen ähnelt der Arbeit von Goldgräbern, welche durch noch näher zu erläuternde Begebenheiten zwar mühselig, zeitraubend, erschöpfend, ja mitunter gefährlich ist, aber deren Ertrag - nämlich die Wiederentdeckung und Wiedererschließung längst verloren geglaubter Literatur - mit Gold kaum aufzuwiegen ist.

Diese Monographie stellt die ausgearbeitete Fassung der Hans-LietzmannVorlesung 2020 dar, welche ich unter dem Titel „Platons Akademie und die Papyri Herkulaneums: Neue Texte durch neue Techniken“ unter den Widrigkeiten der Pandemie in Berlin/Jena (virtuell) halten durfte. Den Organisatoren und Ausrichtern Christoph Markschies und Katharina Bracht sei herzlichst für die Einladung und ihr Wohlwollen gegenüber der Forschung an den Papyri aus Herkulaneum - das Qumran der Klassischen Philologie - gedankt, welches gewiss im interdisziplinären Geiste Hans Lietzmanns gewesen wäre. ${ }^{6}$

The Library of the Villa dei Papiri at Herculaneum (Oxford, 2005); Daniel Delattre, La villa des papyrus et les rouleaux d'Herculanum, la bibliothèque de Philodème (Liège, 2006); Mantha Zarmakoupi (Hg.), The Villa of the Papyri at Herculaneum (Berlin, 2010); Richard Janko, „How to Read and Reconstruct a Herculaneum Papyrus, “ in Ars Edendi Lecture Series, vol. IV (hg. von Barbara Crostini/Gunilla Iversen/Brian Jensen; Toronto, 2016), (117-161); David Blank, „Philodemus“, in Stanford Encyclopedia of Philosophy (hg. von Edward Zalta; online, 2019); Kenneth Lapatin (Hg.), Buried by Vesuvius. The Villa dei Papiri at Herculaneum (Los Angeles, 2019); Francesca Longo Auricchio/Giovanni Indelli/Giuliana Leone/Gianluca Del Mastro, La Villa dei Papiri (Rom, 2020).

6 Die Vorlesung war eine Veranstaltung des Akademienvorhabens (BBAW) „Die alexandrinische und antiochenische Bibelexegese in der Spätantike“ im Zentrum Grundlagenforschung Alte Welt, der Theologischen Fakultät der Humboldt-Universität zu Berlin sowie der Professur für Kirchengeschichte und dem Institut für Altertumswissenschaften der Friedrich-SchillerUniversität Jena. Ferner sei Annette von Stockhausen und Doris Fleischer für die organisatorische Begleitung gedankt. Graziano Ranocchia und Holger Essler haben bei der Erstellung des Buches wertvolle inhaltliche Vorschläge gemacht, für die ich dankbar bin. 